\title{
1 Strigolactones are chemoattractants for host tropism in Orobanchaceae
}

\section{2 parasitic plants}

4 Satoshi Ogawa ${ }^{a}$, Songkui Cui ${ }^{\mathrm{b}}$, Alexandra R.F. White ${ }^{\mathrm{c}}$, David C. Nelson ${ }^{\mathrm{c}}$, Satoko

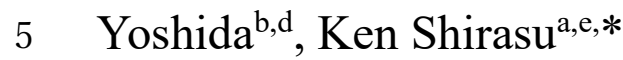

${ }^{a}$ RIKEN Center for Sustainable Resource Science, Yokohama, 230-0045, Japan

$8{ }^{b}$ Division of Biological Science, Graduate School of Science and Technology, Nara Institute of Science

9 and Technology, Ikoma, Nara, 630-0192, Japan

$10{ }^{\mathrm{c} D e p a r t m e n t}$ of Botany and Plant Sciences, University of California, Riverside, CA, 92521, USA

11 dPRESTO, Japan Science and Technology Agency, Kawaguchi, Saitama, 332-0012, Japan

$12{ }^{\mathrm{e}}$ Graduate School of Science, The University of Tokyo, Tokyo, 113-0033, Japan

14 *Author for contact: ken.shirasu@riken.jp 


\section{Abstract}

17 Parasitic plants are worldwide threats that damage major agricultural crops. To initiate infection,

18 parasitic plants have developed the ability to locate hosts and grow towards them. This ability, called

19 host tropism, is critical for parasite survival, but its underlying mechanism remains mostly unresolved.

20 To characterize host tropism, we used the model facultative root parasite Phtheirospermum japonicum,

21 a member of the Orobanchaceae. Here, we show that strigolactones (SLs) function as host-derived

22 chemoattractants. Chemotropism to SLs is also found in Striga hermonthica, a parasitic member of the

23 Orobanchaceae, but not in non-parasites. Intriguingly, chemotropism to SLs in P. japonicum is

24 attenuated in ammonium ion-rich conditions, where SLs are perceived, but the resulting asymmetrical

25 accumulation of the auxin transporter PIN2 is diminished. P. japonicum encodes putative receptors

26 that sense exogenous SLs, whereas expression of a dominant-negative form reduces its chemotropic

ability. We propose a new function for SLs as navigators for parasite roots. 


\section{Introduction}

30 Plant parasitism has independently evolved at least 12 times, accounting for about $1 \%$ of angiosperms

31 or about 4500 species $^{1,2}$. A key trait common to parasitic plants is the ability to connect to host plants and deprive them of nutrients and water, which is often harmful to the hosts ${ }^{3,4}$. Among parasitic plants, some Orobanchaceae species such as Striga spp. and Orobanche spp. are notoriously devastating invaders of major agricultural crops, leading to a multibillion-dollar economic loss annually ${ }^{5,6}$. Most obligate Orobanchaceae parasites have evolved three steps to complete invasion ${ }^{2}$ : germination near the host root in response to host-derived stimulants such as strigolactones (SLs) ${ }^{7}$, active extension of the parasite's root to host roots ${ }^{8,9}$, and connection of the vasculature via formation of an invasive organ called a haustorium ${ }^{2,6,10-12}$. Although many studies have focused on germination and haustorium development, the molecular basis for host tropism is largely unknown. Phtheirospermum japonicum is a facultative hemiparasite in the Orobanchaceae that

parasitism. These studies have revealed the molecular mechanisms regulating haustorium development, 
In angiosperms, $\alpha / \beta$ hydrolases DWARF14 (D14) act as SL receptors ${ }^{21,22}$. After SL

recognition, D14 interacts with an F-box protein MORE AXILLARY GROWTH2 (MAX2), a

component of a SKP1-CULLIN-F-BOX (SCF) E3 ubiquitin ligase complex (SCF ${ }^{\mathrm{MAX} 2}$ ), to mediate SL

signalling ${ }^{23}$. Interestingly, $\mathrm{SCF}^{\mathrm{MAX} 2}$ also mediates signalling driven by karrikins (KARs), a family of

compounds found in smoke that regulate plant growth ${ }^{24,25}$. In Arabidopsis, responses to KARs require

paralogue of $\mathrm{D} 14^{26}$. Whereas KAI2 is a single gene in Arabidopsis, Lamiid species such as Solanales

and Lamiales have duplicated KAI2 genes. KAI2 proteins in Lamiids are categorised into three types:

a "conserved" type found in most Lamiids as well as other angiosperms (KAI2c), an "intermediate"

type only found in Lamiales, including parasites and non-parasites with a few exceptions such as

Orobanche spp. (KAI2i), and a "divergent" type conserved uniquely in Orobanchaceae parasites

$(\text { KAI2d })^{27,28}$. Phylogenetic analyses revealed that Orobanchaceae parasites have rapidly duplicated the

do not require host-derived SLs to germinate also encode multiple KAI2d proteins, suggesting that

KAI2d may have a yet uncovered function distinct from germination stimulants. 
SLs in P. japonicum is negatively regulated by ammonium in the medium, implying that parasites

infect hosts when the nitrogen source is limited. We also describe a functional link between SLs and

the plant hormone, auxin, in chemotropism. Thus, our findings provide a novel function for SLs as

host-produced chemoattractants for parasites.

\section{Results}

\section{Orobanchaceae parasitic plants exhibit chemotropism to SLs}

We previously identified multiple homologues of SL receptor-encoding genes in the genomes of $P$.

japonicum and other Orobanchaceae parasites $^{27}$, although $P$. japonicum does not require exogenous

SLs for germination. Therefore, we hypothesised that $P$. japonicum might use SLs as chemoattractants

rather than for germination. To evaluate this putative function, we devised an experimental system in

which a chemical-soaked filter paper disk was placed next to seedlings. Dimethyl sulfoxide (DMSO)

at a concentration of $0.1 \%(\mathrm{v} / \mathrm{v})$ was used as the negative control. Using this assay system, we

demonstrated that $P$. japonicum exhibited chemotropism to three SLs (rac-strigol (STR), rac-

orobanchol (ORO) and (+)-5-deoxystrigol (5DS)) and two synthetic analogues (rac-GR24 and

83 Yoshimulactone Green $(\mathrm{YLG})^{30}$ ) (Fig. 1a-f, Supplementary Fig. 1, and Supplementary Video 1).

84 Among the tested chemicals, $r a c$-STR and (+)-5DS had the strongest chemotropism-inducing activity

85 at a concentration of $100 \mathrm{nM}$ (Fig. 1g). We also compared the chemotropism activities between 
stereoisomers of 5DS (Supplementary Fig. 1), as the chemical structure of SLs is important for

bioactivities such as germination-inducing activity and hyphal branching-inducing activity ${ }^{31-33}$. In

contrast to (+)-5DS, the unnatural isomer (-)-5DS did not induce chemotropism (Fig. 1h). The

chemoattractant activity of (+)-5DS was comparable to that of (+)-2'-epi-5DS, an SL found in rice ${ }^{34}$,

and stronger than another unnatural isomer (-)-2'-epi-5DS (Fig. 1h). This result suggests that $P$.

japonicum can efficiently sense host-derived natural SLs for chemotropic activity. In addition, using

YLG, a fluorogenic agonist for SL receptors ${ }^{30}$, we observed asymmetrical fluorescence in the root

elongation zone (Fig. 1i), indicating that asymmetrical SL recognition activity induces an asymmetrical

root elongation pattern, leading to chemotropism.

To investigate the chemoattractant activity of SLs on another parasitic plant in the

Orobanchaceae family, we used $S$. hermonthica seedlings just after germination. We found that $S$.

hermonthica exhibited chemotropism to rac-STR and had an asymmetrical YLG recognition pattern

like P. japonicum; however, growth was limited probably due to the lack of sufficient nutrients in the

typically small seeds (Fig. 1j-m). By contrast, a non-parasitic Orobanchaceae Lindenbergia

philippensis exhibited neither chemotropism to rac-STR nor asymmetrical YLG recognition

(Supplementary Fig. 2a,b). Arabidopsis thaliana, a non-parasitic plant in the Brassicaceae, also did

to SLs might be limited to Orobanchaceae parasitic plants.

Next, we conducted infection assays using $P$. japonicum and rice to test the effect of host- 
105 derived SL on host tropism. In agreement with the chemotropism results, more P. japonicum roots

106 grew towards wild-type rice (WT) roots than $d 10$, an SL-deficient mutant that lacks one of the SL-

107 biosynthetic genes ${ }^{35}$ (Fig. 1n-p and Supplementary Video 2). Therefore, we concluded that host108 derived SLs are essential for host tropism in P. japonicum.

Ammonium ions are important for regulating chemotropism

111 Improvement of soil fertility blocks the proliferation of Striga spp. ${ }^{36}$. Thus, we considered that tropic

responses to SLs, one strategy for parasitism, may also be compromised by the presence of abundant

nutrients. To test this hypothesis, we evaluated chemotropism to $r a c$-STR on half-strength Murashige-

114 Skoog (1/2MS) agar with sucrose ${ }^{37}$, a representative nutrient-rich medium on which P.japonicum can

115 grow without parasitism ${ }^{14}$. In contrast to assays on water agar, chemotropism to rac-STR was

116 compromised on 1/2MS agar with sucrose (Fig. 2a,b). We also showed that MS macronutrients

$117\left(\mathrm{KH}_{2} \mathrm{PO}_{4}, \mathrm{KNO}_{3}, \mathrm{NH}_{4} \mathrm{NO}_{3}, \mathrm{CaCl}_{2}\right.$ and $\left.\mathrm{MgSO}_{4}\right)$ with sucrose, but not MS micronutrients, inhibited

118 chemotropism to rac-STR (Fig, 2c,d and Supplementary Fig. 3). In contrast, asymmetrical YLG-

119 derived fluorescence remained in the root elongation zone (Fig. 2e), indicating that nutrients affect the

120 signalling pathway downstream of the SL receptors but not the perception process. To identify the component(s) that impairs chemotropism to SLs, we conducted chemotropism to rac-STR assays using water agar supplemented with individual MS macronutrients. Note that we added $\mathrm{KH}_{2} \mathrm{PO}_{4}$ to nitrogen

$123\left(\mathrm{KNO}_{3}\right.$ and $\left.\mathrm{NH}_{4} \mathrm{NO}_{3}\right)$-containing media in which the nutrient concentration was equal to $1 / 2 \mathrm{MS}$ 
124 because the addition of only a nitrogen source to these media was toxic to P.japonicum. We found that

125 nitrogen, especially ammonium ions, significantly compromised the chemotropic response to $r a c$-STR

126 (Fig. 2f,g). When ammonium ions were omitted from 1/2MS, chemotropism activity was recovered,

127 demonstrating that ammonium ions are necessary and sufficient to impair chemotropism to SLs (Fig.

2h). Next, to investigate downstream signalling, we focused on SUPPRESSOR of MAX2 1 (SMAX1)

genes in P.japonicum that encode homologues of a negative regulator of SL signalling ${ }^{38,39}$. As expected, expression of PjSMAXI was enhanced on 1/2MS and media containing ammonium ions, suggesting

that SL signalling to trigger chemotropism was suppressed (Fig. 2i-k). Overall, our data suggest that

P. japonicum negatively regulates the SL signalling pathway in response to ammonium ions, leading

to a reduction in host tropism capacity.

\section{Auxin response contributes to the tropism to host}

Auxin is a phytohormone well known to regulate tropisms, as exemplified by gravitropism ${ }^{40}$. To test

whether an auxin response also regulates chemotropism to SLs, we used P. japonicum hairy roots transformed with the $3 \mathrm{X}$ mCherry-nuclear localisation signal (NLS) module driven by the auxinresponsive promoter $D R 5^{12,19}$. Asymmetrical activation of the auxin response in root tip epidermal

140 cells was observed in the presence of $\mathrm{rac}$-STR but was diminished on 1/2MS medium (Fig. 3a-h). This

141 STR- and starvation-inducible asymmetrical auxin response coincided with the chemotropism142 exhibiting condition. We further focused on PIN-FORMED2 (PIN2), one of the PIN-family auxin 
143 efflux transporters ${ }^{41}$, as PIN2 plays a major role in root tropisms ${ }^{42}$ and PjPIN2 is a representative PIN

144 localised in P. japonicum root epidermal cells ${ }^{19}$. Using the PjPIN2-Venus module driven by the native

145 promoter, we found an asymmetrical increase in PjPIN2 accumulation in epidermal cells of the root

146 elongation zone in water agar (Fig. 3i, j, m, n), but not in a medium containing 1/2MS+suc (Fig. 3k, 1 ,

$147 \mathrm{o}, \mathrm{p})$. These results suggest that local SL perception leads to local auxin accumulation for tropism,

148 likely via PIN2, and that the nutrient-based inhibition of chemotropism occurs between SL perception

149 and PIN2 accumulation.

\section{KAI2d proteins are the SL receptors involved in chemotropism}

152 Next, we investigated if KAI2d proteins are involved in chemotropism. Using KAI2 protein sequences

153 found in land plants, we constructed phylogenetic trees. Seven candidate KAI2d homologues encoded

154 in the P. japonicum genome ${ }^{16}$ separated into two groups: the first group is similar to KAI2d found in

155 obligate hemiparasites such as $S$. hermonthica, and the second group is similar to those in obligate

156 holoparasites, such as Orobanche spp. (Fig. 4a and Supplementary Fig. 4). In combination with

157 transcriptome data in P. japonicum ${ }^{18}$, we found that the gene expression levels of PjKAI2d2, PjKAI2d3

158 and PjKAI2d3.2, the $K A I 2 d$ homologues most similar to those in obligate hemiparasites, were high in

159 seedlings. In contrast, little expression was observed in the roots post-infection. This pattern was

160 consistent with the finding that the majority of $K A I 2 d$ genes in $S$. hermonthica were highly expressed

161 in seedlings (Fig. 4a) ${ }^{29}$. Next, we investigated the effects of $r a c$-STR and nutrient conditions on KAI2 
expression in P. japonicum. Of the 7 PjKAI2d genes as well as PjKAI2i and PjKAI2c, only PjKAI2d2 and PjKAI2d3.2 showed relatively high expression levels in basal conditions and were further increased by rac-STR treatment. Basal and induced expression of these genes was attenuated in the

nutrient-rich 1/2MS condition (Fig. 4b). Although nutrients also suppressed PjKAI2d3 expression, the

gene was not upregulated by rac-STR, unlike PjKAI2d2 or PjKAI2d3.2. The reason why PjKAI2d3

had a different expression pattern from previous transcriptome data might be because PjKAI2d3.2 transcripts may have been mistakenly mapped to $P j K A I 2 d 3$ due to their high degree of conservation

169 (96\% amino acid identity). To test the SL-responding ability of PjKAI2d2, PjKAI2d3 and PjKAI2d3.2,

we adopted a modified cross-species complementation method, which had been successful in previous studies $^{27,43,44}$. We used the $A$. thaliana 114 kai2 double mutant in the Col-0 background ${ }^{45}$ and evaluated responses to $\mathrm{rac}$-STR by seed germination rates (Fig. 4c). Since Col-0 seeds are known to lose their primary dormancy rapidly after maturation ${ }^{28}$, we stratified seeds at $4{ }^{\circ} \mathrm{C}$ overnight after sowing to break dormancy and, therefore, to exclude the effect of dormancy. Germination phenotypes in the control AtKAI2-complemented lines were comparable to those in the wild-type Col-0 without significant changes resulting from rac-STR treatment, although D14 was still missing. Concurrently, rac-STR promoted germination in PjKAI2d2- or PjKAI2d3.2-introduced lines with basal germination rates comparable to those of $d 14$ kai2 (Fig. 4c). These data indicate that at least PjKAI2d and PjKAI2d3.2 are functional as receptors of exogenous SLs. 
by gene editing would be desirable; however, $K A I 2 d$ genes are multicopy and likely to be functionally

redundant (Supplementary Fig. 5). As we cannot yet create transgenerational P.japonicum transgenics,

183 it is difficult to edit or knockdown multicopy genes ${ }^{46}$. Hence, we set out to generate dominant-negative

plants by overexpressing the substituted KAI2d2, in which the functions of native KAI2d proteins are impeded. KAI2d proteins have a highly conserved arginine that is also conserved in D14 in $A$. thaliana

and rice (Supplementary Fig. 5). Substitution of this arginine in D14 of $A$. thaliana and rice with

histidine did not interfere with the hydrase activity; however, introducing the substituted D14 into each

mutant did not complement the phenotypes due to loss of protein-protein interaction ${ }^{47}$. Therefore, we

considered this substitution appropriate for generating KAI2d dominant-negative plants. We

transformed P. japonicum seedlings with the substituted PjKAI2d2 (PjKAI2d2 $\left.{ }^{R 183 H}\right)$ driven by the constitutively active promoter $P j A C T^{17}$. Using the resulting transgenic hairy roots, we tested chemotropism to rac-STR and quantified the expression levels of PjSMAX1.2. Overexpression of

PjKAI2d2 $2^{R 183 H}$ hindered chemotropism to rac-STR, indicating that $P j K A I 2 d 2^{R 183 H}$-overexpressing

194 hairy roots were dominant negative and that disturbing the interaction of KAI2d with the partner(s)

195 was likely to cause loss of chemotropism to SLs (Fig. 4d). In addition, the expression level of 196 PjSMAX1.2 was constitutively enhanced in the dominant-negative plants (Fig. 4e), suggesting 197 suppression of SL signalling. Taken together, our results indicate that PjKAI2d proteins play an 198 important role in SL signalling, leading to chemotropism. 


\section{Discussion}

201 Obligate parasitic plants in the Orobanchaceae must attach to host roots within several days after

202 germination to acquire nutrients and water because resources in their seeds are limited, and they cannot

203 obtain sufficient energy for their survival by photosynthesis. Finding a host root to parasitise is also

204 important for facultative parasites, which can survive without a host, as parasitisation significantly

enhances its growth ${ }^{14,48}$. Thus, efficient growth towards host roots is critical for facultative and obligate

Orobanchaceae parasites. Our study shows that both facultative $P$. japonicum and obligate $S$.

hermonthica use SLs as chemoattractants, leading to host tropism (Fig. 1). As SLs do not induce

chemotropism in L. philippensis, a non-parasitic member of the Orobanchaceae (Supplementary Fig.

2), this chemotropism phenotype may have been acquired during parasite evolution. Intriguingly, the

germ tube of Orobanche cumana, a holoparasite, is guided by costunolide, a sunflower-derived

sesquiterpene lactone, but not by GR24, a synthetic SL analogue ${ }^{49}$. In comparison, O. cumana

germination is stimulated by costunolide and GR2 $24^{27,49}$. Therefore, it is likely that the nature of

chemoattractants is different among phylogenetic clades $^{2}$, in contrast with the commonality of using

214 SLs as germination stimulants in obligate parasites. Host preferences of Striga spp. ${ }^{3}$ and Orobanche

spp. ${ }^{50}$ may be reflected by differences in their chemoattractants. Future identification of specific

chemoattractants produced by each host may uncover the chemical basis for host preference in

Orobanchaceae parasites. 
219 establish symbiotic relationships with plants to exchange soil-derived nutrients such as phosphate and

220 nitrogen with plant-derived carbon sources ${ }^{52,53}$. Interestingly, D14L, a KAI2 homologue, is required

221 for AMF symbiosis in rice ${ }^{54}$. D14L derepresses downstream signalling by removing the suppressor

222 SMAX1, resulting in elevated AMF colonization ${ }^{39}$. In our study of $P$. japonicum, we found that

223 disturbance of KAI2d function(s) by overexpressing a substituted KAI2d2 resulted in elevated

224 PjSMAX1.2 expression and loss of chemotropism to SLs (Fig. 4d,e). As KAI2d-mediated SL

225 perception for chemotropism is important in host infection to obtain nutrients, it is tempting to think

that Orobanchaceae parasites may have converted the KAI2-based symbiotic AMF communication

tool via MAX2 into a parasitic host-detection system. In this context, the observation that

chemotropism to SLs occurs only in nitrogen-deficient conditions is intriguing (Fig. 2). Plants often

229 produce and exude SLs when nitrogen is deficient for attracting $\mathrm{AMF}^{55}$, which can, in turn, promote

230 plant nitrogen acquisition. Indeed, in the case of rice, $\sim 40 \%$ of the plant's nitrogen requirement can be

231 acquired by $\mathrm{AMF}^{56}$. It is possible that, in Orobanchaceae parasites, the primary nitrogen source may

232 have shifted from AMF to host plants, potentially by evolving KAI2-mediated signalling via MAX2 ${ }^{57}$

233 Consistently, P. japonicum effectively transfers nitrogen from hosts, especially in nutrient-deficient

234 conditions $^{58}$. AMF colonisation in P. japonicum has not been reported, but some Pedicularis species,

235 facultative hemiparasitic plants in the Orobanchaceae, can accommodate $\mathrm{AMF}^{59}$. How such parasites

236 can coordinate SLs and KAI2/KAI2d proteins to accommodate both AMF and hosts is an interesting

237 question to be answered. Future genomic surveys of the AMF-accommodating abilities in 
238 Orobanchaceae plants may provide molecular clues of how the KAI2/KAI2d-based symbiotic-parasite

239 relationship has evolved.

Complementation of the $d 14$ kai2 mutant with $P j K A I 2 d 2$ or PjKAI2d3.2 partially rescued seed

germination in an exogenous SL-dependent manner (Fig. 4c). This result indicates that SL-bound

PjKAI2d2 and PjKAI2d3.2 may not efficiently bind to A. thaliana MAX2 and/or SMAX1. In addition,

PjKAI2d2 and PjKAI2d3.2 failed to complement the germination-attenuated phenotype without SL

application, indicating that these proteins are unlikely to bind to unidentified endogenous KAI2 ligands

(KLs) to activate downstream signalling. Similarly, S. hermonthica KAI2c, KAI2i, KAI2d1 and KAI2d2

do not complement the $A$. thaliana kai2 mutant without $\mathrm{SLs}^{27}$, suggesting that $S$. hermonthica may

have lost KL-sensing activity for germination. Further characterisation of various KAI2 proteins and

associated proteins in the Orobanchaceae family and other land plants will reveal signalling

specificities differentiating chemotropism and germination functions. This research direction may

eventually provide clues of how neo-functionalisation of KAI2/KAI2d occurs in plants.

(Fig. 3). This observation provides a functional link between SL and auxin signalling in the host

tropism of P. japonicum. To date, the effects of SLs on auxin-mediated processes have been studied 
257 canalisation $^{62}$. In roots, asymmetrical PIN2 accumulation in epidermal cells is a key factor in root

258 bending, as seen in gravitropism ${ }^{62}$. In P. japonicum, exogenous SLs are perceived by the epidermal

259 cells in the elongation zone, as indicated by YLG-based fluorescence, where PjPIN2 accumulates (Fig.

$2601 \mathrm{i}$ and $3 \mathrm{n}$ ). This result is consistent with the finding that KAI2 is required for the local accumulation

261 of PIN2 in $A$. thaliana roots $^{63}$. As A. thaliana does not exhibit chemotropism to SLs (Supplementary

262 Fig. 2c,d) and frequently SLs are evenly distributed throughout $A$. thaliana media, it is difficult to

263 compare the effects of SLs on PIN2 accumulation in A. thaliana and P. japonicum. An alteration in

264 SL-PIN2 relationships may have occurred in Orobanchaceae parasites that enabled chemotropism.

265 Importantly, SLs can be perceived in nutrient-rich conditions (Fig. 2e), but the asymmetrical PIN2

accumulation occurs only in nitrogen-, especially ammonium ion-, deficient conditions (Fig. $3 \mathrm{n}$ and

$3 p)$. The SL signalling pathway is also affected by ammonium ions, as indicated by upregulation of

SMAX1.1 and SMAX1.2 (Fig. 2i-k). This finding suggests that ammonium ions regulate root tropism

by controlling PIN2 accumulation potentially via SMAX regulation. As rice roots exhibit

chemotropism to ammonium ions ${ }^{64}$, it would be interesting to test whether that tropism to ammonium

ions also involves an asymmetric accumulation of PIN2, which may be regulated by SL signalling in

rice.

In summary, our study unveils important molecular clues about host tropism in Orobanchaceae

274 parasitic plants, providing a novel function of SLs as chemoattractants. We expect this study will 
276 infection in parasitic plants. Such future studies will help design solutions for protecting agricultural

277 fields from nuisance weeds.

279 Methods

280

281

Plant materials and growth conditions

Unless otherwise noted, P. japonicum (Thunb.) Kanitz seeds and A. thaliana seeds (Col-0, d14 kai2 ${ }^{45}$

and complemented lines) were germinated on $1 / 2 \mathrm{MS}$ medium $(0.8 \%(\mathrm{w} / \mathrm{v})$ INA agar, $\mathrm{pH} 5.8)$

containing $1 \%(\mathrm{w} / \mathrm{v})$ sucrose. Before sowing, seeds were sterilised with a diluted commercial bleach

solution (Kao, Tokyo, Japan, 10\% (v/v) for P. japonicum and 5\% (v/v) for A. thaliana) for 5 minutes and rinsed at least 5 times with sterilised water. Plate-sown seeds were stratified at $4{ }^{\circ} \mathrm{C}$ in the dark for

1 to 3 night(s), then grown horizontally in long-day conditions (16-h light $\left(\sim 40\right.$ and $\sim 30 \mu \mathrm{mol} \mathrm{m}^{-2} \mathrm{~s}^{-1}$

for P. japonicum and A. thaliana, respectively), 8-h dark at $25{ }^{\circ} \mathrm{C}$ and $22{ }^{\circ} \mathrm{C}$ for P. japonicum and $A$.

thaliana, respectively). $P$. japonicum and $A$. thaliana seeds were grown at $70 \%$ and $50 \%$ humidity,

respectively. $S$. hermonthica seeds were carefully handled with glass pipettes as the seeds stick to

vortex, then sterilised with a fresh bleach solution for 5 minutes, followed by at least 5 rinses with

sterilised water. Seeds were then soaked in 5-mL of sterilised water in 6-well plates and incubated in 
295 (STR $)^{65}$ solution, and the plates were incubated for 4 hours in the dark at $25^{\circ} \mathrm{C}$. The (+)-STR solution

296 was then replaced with water, and the plates were incubated at least 24 hours before further analysis

297 to exclude the residual effects of (+)-STR. L. philippensis seeds were sterilised with a diluted 298 commercial bleach solution (Kao, Tokyo, Japan, 5\% (v/v)) for 5 minutes and rinsed at least 5 times 299 with sterilised water before soaking in 5-mL of sterilised water in 6-well plates. Water-soaked seeds 300 were grown in long-day conditions $\left(\sim 30 \mu \mathrm{mol} \mathrm{m}^{-2} \mathrm{~s}^{-1}\right)$ at $22{ }^{\circ} \mathrm{C}$ for six days. For further analyses, seedlings were carefully handled with glass pipettes and a pair of tweezers. $O$. sativa (japonica, c.v. 302 Shiokari) seeds of WT and $d 10^{35}$ were sterilised with $70 \%(\mathrm{v} / \mathrm{v})$ ethanol for 3 minutes, followed by a 303 diluted commercial bleach solution (Kao, Tokyo, Japan, 50\% (v/v)) for 30 minutes and rinsed with sterilized water at least 5 times. Surface-sterilized seeds were then grown vertically on $0.6 \%(\mathrm{w} / \mathrm{v})$ water agar in long-day conditions $\left(18 \mathrm{~h} / 6 \mathrm{~h}\right.$ light/dark, $\left.\sim 120 \mu \mathrm{mol} \mathrm{m}{ }^{-2} \mathrm{~s}^{-1}\right)$ at $25^{\circ} \mathrm{C}$.

\section{Chemicals}

SLs and analogues used in this study are listed in Supplementary Table 1. Chemicals were stored at -

\section{Chemotropism assays}

312 For assays using P. japonicum seedlings, 3-day-old seedlings were transferred carefully to solid media 
314 agar, $\mathrm{pH}$ 5.8), incubated vertically for 1 day, and transferred to the media used for the assays. Filter

315 paper disks (4-mm diameter) were soaked with the chemical-containing solution before being placed

316 5-mm from the seedlings. P. japonicum transgenic hairy roots were generated from seedlings 3- to 4-

317 weeks post-transformation and were transferred carefully to solid medium without nutrients $(0.7 \%$

318 (w/v) INA agar, $\mathrm{pH} 5.8$ ), incubated horizontally for 2 days, and transferred to new solid medium

319 without nutrients. Filter paper disks (4-mm diameter) were soaked with a chemical-containing solution

320 before being placed 5-mm from the seedlings. For assays using $S$. hermonthica, germinated seedlings were transferred carefully to solid medium without nutrients $(0.7 \%$ INA agar $(\mathrm{w} / \mathrm{v}), \mathrm{pH} 5.8)$. Filter

paper disks (4-mm diameter) were soaked with a chemical-containing solution, before being placed 3-

$\mathrm{mm}$ from the seedlings. For assays using L. philippensis, germinated seedlings were transferred

carefully to solid medium without nutrients $(0.7 \%$ INA agar (w/v), $\mathrm{pH} 5.8)$. Filter paper disks (4-mm

diameter) were soaked with a chemical-containing solution, before being placed 3-mm from the

seedlings. For assays using A. thaliana, 2-day-old seedlings were transferred carefully to solid medium

without nutrients $(0.7 \%$ (w/v) INA agar, $\mathrm{pH} 5.8)$, incubated vertically for 1 day, and transferred to new

solid medium. Filter paper disks (4-mm diameter) were soaked with a chemical-containing solution,

before being placed 5-mm from the seedlings. We defined seedlings that exhibited chemotropism as 
333 For fluorescence microscopy, photos were taken using a fluorescence stereo microscope (M165 FC,

334 Leica) before and after chemical-treated seedlings were incubated in the dark at $70 \%$ humidity for 1

335 day, $25{ }^{\circ} \mathrm{C}$ for $P$. japonicum and $S$. hermonthica, and $22{ }^{\circ} \mathrm{C}$ for A. thaliana and L. philippensis,

respectively, except for time-lapse microscopy. For time-lapse microscopy, 100 photos were taken

throughout 18 hours using a fluorescence stereo microscope (M205 FA, Leica) in the dark at $25{ }^{\circ} \mathrm{C}$.

338 For confocal microscopy, photos were taken using an inverted confocal microscope (TCS SP5 II,

Leica). Fluorescein from hydrolysed YLG was excited with a 488-nm laser, and the detected emission

spectra were observed at 500-531nm. Excitation and detection of Venus and mCherry fluorescence using Image $\mathrm{J}^{66}$.

\section{Host chemotropism assay for $P$. japonicum}

P. japonicum seeds were sterilised, sown, stratified, and grown on solid growth medium containing $1 / 2$

MS, $0.6 \%(\mathrm{w} / \mathrm{v})$ agar, and $1 \%$ sucrose (pH 5.8) for 3 days in the dark at $25^{\circ} \mathrm{C}$. The seedlings were then

transferred to $0.6 \%(\mathrm{w} / \mathrm{v})$ agar medium without nutrients and grown vertically for 1 day in long-day

conditions at $25^{\circ} \mathrm{C}$. Rice seeds were sterilised, sown, and grown for 4 days as described in the Plant

materials and growth conditions section. Host chemotropism assays were then performed on $0.6 \%$ 
352 Single roots of similar length from each genotype were chosen and aligned vertically in parallel at a

353 distance of approximately $2 \mathrm{~mm}$. A P. japonicum seedling was then placed between two rice roots and

a single $P$. japonicum root was carefully placed parallel to the middle of the rice roots. Relatively young

root regions (approximately $500 \mathrm{~mm}$ from the tip) of the host were chosen for the initial infection

position ( 0 days) because these areas produce few to no lateral roots during the observation period that

would otherwise physically disturb P. japonicum roots from bending. Three independent experiments

were conducted with each replicate containing 7 pairs. For about half of the pairs, WT seedlings were

placed at the left and $d 10$ seedlings at the right, and for the rest vice versa. To keep the roots on the

medium, a cover glass was placed on the top of the roots. The plates were sealed with surgical tape

and positioned vertically in the growth chamber. Images were captured 0,1 , and 2 day(s) after infection

using a wide zoom stereo microscope (Olympus SZX16).

ImageJ (version 1.52q), Microsoft Excel and Adobe Illustrator software were employed to quantify

364 the images. In all cases, WT roots were positioned on the left and $d 10$ was on the right. First, images

365 derived from 1- or 2-day(s) post-infection were superimposed onto their corresponding 0-day images

using Adobe Illustrator. Next, using ImageJ, the position of the P. japonicum root tip was defined as y

$=0$ and the position of the left (WT) host at horizontal axis with the $P$. japonicum root tip was defined

as $\mathrm{x}=0$ in 0 -day samples. Thus, each rice root and the $P$. japonicum root tip were assigned a unique $\mathrm{x}$

and y value: for instance, WT rice $(\mathrm{x}=0 \mu \mathrm{m}, \mathrm{y}=0 \mu \mathrm{m}), P$.japonicum $(\mathrm{x}=904.8 \mu \mathrm{m}, \mathrm{y}=0 \mu \mathrm{m})$ and

$d 10$ rice $(\mathrm{x}=1979.6 \mu \mathrm{m}, \mathrm{y}=0 \mu \mathrm{m})$ at 0 day. The $\mathrm{xy}$ positions of the P. japonicum root tip at 1 - and 2- 
371 day(s) post infection relative to 0 day were then measured. Raw xy data generated from all samples as

372 described above were subjected to centring and scaling processes before generating the graphs shown

373 in the figures (For details refer to Source Data).

\section{Extraction of total RNA and RT-qPCR}

376 To extract total RNA from P. japonicum seedlings, 3-day-old seedlings were transferred to media containing varying nutrients and were grown vertically for 1 day. The seedlings were transferred again

to the same medium used for the assays. At 3 hours after incubation, root tips were excised and immediately frozen in liquid nitrogen. To extract total RNA from P. japonicum transgenic hairy roots,

root tips of the generated hairy roots were excised and immediately frozen in liquid nitrogen after the chemotropism assays (see Chemotropism assay section). Total RNA extraction, cDNA synthesis and

RT-qPCR were performed as previously described ${ }^{12}$.

\section{Cloning}

385 The constructs $p D R 5:: 3 x m$ Cherry-NLS and pPjPIN2::PjPIN2-Venus ${ }^{19}$ were previously described.

pKAI2pro-GW ${ }^{67}$ was used as the Gateway destination vector to express each protein under the control

of the AtKAI2 promoter. The AtKAI2-expressing vector pKAI2-AtKAI2 was previously described ${ }^{67}$.

PjKAI2d-expressing vectors were constructed as follows: the genomic regions containing each coding 
390 was PCR amplified with primers containing attB sites, subcloned into pDONR/Zeo (Thermo Fisher

391 Scientific) by Gateway BP cloning (Thermo Fisher Scientific) to generate entry vectors, then cloned

392 into pKAI2pro-GW by Gateway LR cloning (Thermo Fisher Scientific), yielding pKAI2-PjKAI2d2,

393 pKAI2-PjKAI2d3 and pKAI2-PjKAI2d3.2. For constructing the expression vectors to transform $P$.

394 japonicum, we used GoldenGate technology for assembling modules ${ }^{68}$. The GoldenGate modules

395 containing the $35 S$ promoter $(p 35 S$, pICH51266) or $35 S$ terminator $(35 S t$, pICH41414) were pre-

396 existing $^{68}$. Modules containing the actin promoter ( $\left.p P j A C T\right), 3 \times$ Venus-NLS CDS or HSP 18.2

397 terminator with the $3^{\prime}$-untranslated region (HSPt) were described previously ${ }^{13,17}$. A single mutation

398 was introduced into the PjKAI2d2 CDS using a KOD plus Mutagenesis kit (TOYOBO) with the entry

399 vector described above as a template. The mutated PjKAI2d2 CDS was PCR amplified and cloned into

400 pICH41308 to generate a level 0 CDS1 module. The modules were assembled and cloned into level 1

401 vectors to generate $p P j A C T:: P j K A I 2 d 2^{R 183 H}: H S P t$ and $p 35 S:: 3 x v e n u s-N L S: 35 S t$ transcription units.

402 The resulting transcription units were further combined and cloned into the level 2 binary vector

403 pAGM4723. End-linkers and dummies were used as needed.

404

405

Transformation of $P$. japonicum

406 Agrobacterium rhizogenes AR1193 strain was used to transform P. japonicum seedlings. A.

407 rhizogenes-mediated transgenic hairy roots were generated and identified according to previously

408 described methods ${ }^{12,13}$. 


\section{Phylogenetic analyses}

411 We used the CLC Main Workbench (ver. 8.0, Qiagen) for phylogenetic analyses. The CDSs of KAI2

412 used for the phylogenetic analyses in Conn et al. ${ }^{27}$ were automatically translated and used in our study.

413 Two candidate genes, KAI2d3.2 and KAI2d4.2 in P. japonicum, which were newly identified from

414 the P. japonicum genome by BLASTp analysis using PjKAI2d1-PjKAI2d5 as queries (with e-values

415 under $1 \mathrm{e}^{-100}$ ), were added to the KAI2 sequence group. The KAI2 sequences processed by trimAL

$416 \mathrm{v} 1.2^{69}$ using the automated 1 settings were aligned using default settings. After alignment, phylogenetic

417 trees were drawn using the maximum-likelihood method with 1,000 bootstrap repetitions. We

418 generated the figures using iTOL v6 (https://itol.embl.de/ ${ }^{70}$ ).

\section{Transformation of $\boldsymbol{A}$. thaliana and cross-species complementation assays}

pKAI2-AtKAI2, pKAI2-PjKAI2d2, pKAI2-PjKAI2d3 or pKAI2-PjKAI2d3.2 were electroporated

into Agrobacterium tumefaciens GV3101 competent cells. The resulting bacterial cells were used to

inoculate flowers in a transformation method modified from Martinez-Trujillo et al. ${ }^{71}$. For germination

assays, $A$. thaliana seeds that were at least 1-month old were used. Seeds were sterilised with chlorine

gas for 2-3 hours in a safety cabinet and dried for at least 2 hours in a clean bench, followed by sowing

on solid media without nutrients $(0.7 \%(\mathrm{w} / \mathrm{v})$ INA agar, $\mathrm{pH} 5.8)$ containing $1 \mu \mathrm{M}$ rac-STR or $0.1 \%$

(v/v) DMSO. Plate-sown seeds were stratified at $4{ }^{\circ} \mathrm{C}$ in the dark for 1 night, then incubated 
428 horizontally in the dark. Germination rates, defined by radicle emergence, were scored periodically.

$430 \quad$ Statistical analyses

431 Welch's $t$ test and two-way ANOVA, Tukey's multiple comparison test were performed in Microsoft

432 Excel 2016 and GraphPad Prism version 8.1, respectively. Details of statistical analyses including 433 statistical methods, numbers of individual batches, numbers of technical replicates, numbers of 434 biological replicates, and statistical significances are described in each figure legend.

\section{Data and code availability}

437 Transcriptome data for S. hermonthica ${ }^{29}$ and $P$. japonicum $^{18}$ are available from the DNA Data Bank 438 of Japan (http://www.ddbj.nig.ac.jp/) under accession numbers DRA008615 and DRA003608 for $S$.

439 hermonthica and DRA010010 for P. japonicum, respectively. Sequence data from this study have

440 been deposited at the GenBank/EMBL libraries and are publicly available as of the date of 441 publication. Accession numbers are provided in Supplementary Table 2. Gene IDs of the $P$.

442 japonicum genes investigated in this study are provided in Supplementary Table 2. Source data are 443 provided with this manuscript.

446 1. Westwood, J. H., Yoder, J. I., Timko, M. P. \& dePamphilis, C. W. The evolution of parasitism 
in plants. Trends Plant Sci 15, 227-235 (2010).

448 2. Mutuku, J. M., Cui, S., Yoshida, S. \& Shirasu, K. Orobanchaceae parasite-host interactions.

New Phytol 230, 46-59 (2021).

3. Spallek, T., Mutuku, M. \& Shirasu, K. The genus Striga: a witch profile. Mol Plant Pathol 14, $861-869(2013)$

between parasitic plants and their hosts. Annu Rev Phytopathol 57, 279-299 (2019).

Pest Manag Sci 65, 453-459 (2009). in parasitic plants. Annu Rev Plant Biol 67, 643-667 (2016). (1966).

8. Williams, C. N. Growth and morphogenesis of Striga seedlings. Nature 189, 378-381 (1961).

$4629 . \quad$ Yoshida, S. \& Shirasu, K. Multiple layers of incompatibility to the parasitic witchweed, Striga hermonthica. New Phytol 183, 180-189 (2009).

10. Cui, S. et al. Haustorial hairs are specialized root hairs that support parasitism in the facultative parasitic plant Phtheirospermum japonicum. Plant Physiol 170, 1492-1503 (2016). 
466 11. Masumoto, N. et al. Three-dimensional reconstructions of haustoria in two parasitic plant species in the Orobanchaceae. Plant Physiol 185, 1429-1442 (2021).

12. Ogawa, S. et al. Subtilase activity in intrusive cells mediates haustorium maturation in parasitic plants. Plant Physiol 185, 1381-1394 (2021).

13. Ishida, J. K. et al. Local auxin biosynthesis mediated by a YUCCA flavin monooxygenase

14. Spallek, T. et al. Interspecies hormonal control of host root morphology by parasitic plants.

15. Ishida, J. K., Yoshida, S., Ito, M., Namba, S. \& Shirasu, K. Agrobacterium rhizogenes-mediated

16. Cui, S. et al. Ethylene signaling mediates host invasion by parasitic plants. Sci Adv 6, abc2385 (2020).

17. Wakatake, T., Yoshida, S. \& Shirasu, K. Induced cell fate transitions at multiple cell layers configure haustorium development in parasitic plants. Development 145, dev164848 (2018).

18. Kurotani, K. I. et al. Host-parasite tissue adhesion by a secreted type of $\beta$-1,4-glucanase in the parasitic plant Phtheirospermum japonicum. Commun Biol 3, 407 (2020).

19. Wakatake, T., Ogawa, S., Yoshida, S. \& Shirasu, K. An auxin transport network underlies xylem bridge formation between the hemi-parasitic plant Phtheirospermum japonicum and 
host Arabidopsis. Development 147, dev187781 (2020).

20. Greifenhagen, A. et al. The Phtheirospermum japonicum isopentenyltransferase PjIPT1a regulates host cytokinin responses in Arabidopsis. New Phytol 232, 1582-1590 (2021). branching hormone, strigolactone. Curr Biol 22, 2032-2036 (2012). and other small signals. New Phytol 225, 621-636 (2020). of KAI2 paralogues. BMC Biol 15, 52 (2017). in Arabidopsis thaliana. Proc Natl Acad Sci U S A 108, 8897-8902 (2011).

25. Khosla, A. et al. Structure-function analysis of SMAX1 reveals domains that mediate its

24. Nelson, D. C. et al. F-box protein MAX2 has dual roles in karrikin and strigolactone signaling

23. Bythell-Douglas, R. et al. Evolution of strigolactone receptors by gradual neo-functionalization (2020).

26. Waters, M. T. et al. Specialisation within the DWARF14 protein family confers distinct responses to karrikins and strigolactones in Arabidopsis. Development 139, 1285-1295 (2012).

27. Conn, C. E. et al. Convergent evolution of strigolactone perception enabled host detection in parasitic plants. Science 349, 540-543 (2015). 
Physiol 185, 1353-1373 (2021).

29. Yoshida, S. et al. Genome sequence of Striga asiatica provides insight into the evolution of plant parasitism. Curr Biol 29, 3041-3052 (2019).

30. Tsuchiya, Y. et al. Probing strigolactone receptors in Striga hermonthica with fluorescence.

Science 349, 864-868 (2015).

for hyphal branching in AM fungi. Plant Cell Physiol 51, 1104-1117 (2010).

511 32. Nomura, S., Nakashima, H., Mizutani, M., Takikawa, H. \& Sugimoto, Y. Structural requirements of strigolactones for germination induction and inhibition of Striga gesnerioides seeds. Plant Cell Rep 32, 829-838 (2013).

33. Scaffidi, A. et al. Strigolactone hormones and their stereoisomers signal through two related receptor proteins to induce different physiological responses in Arabidopsis. Plant Physiol 165, 1221-1232 (2014) tobacco. Mol Plant 6, 153-163 (2013).

35. Umehara, M. et al. Inhibition of shoot branching by new terpenoid plant hormones. Nature $\mathbf{4 5 5}$, 195-200 (2008).

Physiol 185, 1339-1352 (2021). 
523 37. Murashige, T. \& Skoog, F. A revised medium for rapid growth and bio assays with tobacco tissue cultures. Physiol Plant 15, 473-497 (1962).

38. Walker, C. H., Siu-Ting, K., Taylor, A., O'Connell, M. J. \& Bennett, T. Strigolactone synthesis

39. Choi, J. et al. The negative regulator SMAX1 controls mycorrhizal symbiosis and strigolactone biosynthesis in rice. Nat Commun 11, 2114 (2020).

40. Leyser, O. Auxin signaling. Plant Physiol 176, 465-479 (2018).

41. Wisniewska, J. et al. Polar PIN localization directs auxin flow in plants. Science 312, 883 (2006).

42. Zhang, Y., Xiao, G., Wang, X., Zhang, X. \& Friml, J. Evolution of fast root gravitropism in

43. Toh, S. et al. Structure-function analysis identifies highly sensitive strigolactone receptors in

45. Li, W. et al. Comparative functional analyses of DWARF14 and KARRIKIN INSENSITIVE 2 in drought adaptation of Arabidopsis thaliana. Plant J 103, 111-127 (2020).

46. Laohavisit, A. et al. Quinone perception in plants via leucine-rich-repeat receptor-like kinases. 
Nature 587, 92-97 (2020).

543 47. Seto, Y. et al. Strigolactone perception and deactivation by a hydrolase receptor DWARF14.

Nat Commun 10, 191 (2019).

48. Seel, W. E. \& Jeschke, W. D. Simultaneous collection of xylem sap from Rhinanthus minor

of attachment. New Phytol 143, 281-298 (1999).

49. Krupp, A., Bertsch, B. \& Spring, O. Costunolide influences germ tube orientation in sunflower broomrape - a first step toward understanding chemotropism. Front Plant Sci 12, 699068 (2021).

53. Kobae, Y. et al. Strigolactone biosynthesis genes of rice are required for the punctual entry of

52. Govindarajulu, M. et al. Nitrogen transfer in the arbuscular mycorrhizal symbiosis. Nature 435, 819-823 (2005). arbuscular mycorrhizal fungi into the roots. Plant Cell Physiol 59, 544-553 (2018).

54. Gutjahr, C. et al. Rice perception of symbiotic arbuscular mycorrhizal fungi requires the 
karrikin receptor complex. Science 350, 1521-1524 (2015).

562 55. Yoneyama, K. et al. How do nitrogen and phosphorus deficiencies affect strigolactone production and exudation? Planta 235, 1197-1207 (2012).

564 56. Wang, S. et al. Functional analysis of the OsNPF4.5 nitrate transporter reveals a conserved mycorrhizal pathway of nitrogen acquisition in plants. Proc Natl Acad Sci U S A 117, 1664916659 (2020).

57. Hull, R., Choi, J. \& Paszkowski, U. Conditioning plants for arbuscular mycorrhizal symbiosis through DWARF14-LIKE signalling. Curr Opin Plant Biol 62, 102071 (2021).

58. Irving, L. J. et al. Host nutrient supply affects the interaction between the hemiparasite Phtheirospermum japonicum and its host Medicago sativa. Environ Exp Bot 162, 125-132 (2019).

59. Li, A. R. \& Guan, K. Y. Arbuscular mycorrhizal fungi may serve as another nutrient strategy (2008).

60. Brewer, P. B., Koltai, H. \& Beveridge, C. A. Diverse roles of strigolactones in plant development. Mol Plant 6, 18-28 (2013).

61. Shinohara, N., Taylor, C. \& Leyser, O. Strigolactone can promote or inhibit shoot branching by triggering rapid depletion of the auxin efflux protein PIN1 from the plasma membrane. PLoS

Biol 11, e1001474 (2013). 
580 62. Zhang, J. et al. Strigolactones inhibit auxin feedback on PIN-dependent auxin transport canalization. Nat Commun 11, 3508 (2020).

63. Villaécija-Aguilar, J. A. et al. KAI2 promotes Arabidopsis root hair elongation at low external phosphate by controlling local accumulation of AUX1 and PIN2. Curr Biol 32, 228-236 (2022).

source. Plant Cell Physiol 61, 546-553 (2020).

65. Hirayama, K. \& Mori, K. Synthesis of $(+)-$ Strigol and $(+)$-Orobanchol, the germination

69. Capella-Gutiérrez, S., Silla-Martínez, J. M. \& Gabaldón, T. trimAl: a tool for automated analysis. Nat Methods 9, 671-675 (2012).

67. Waters, M. T., Scaffidi, A., Flematti, G. \& Smith, S. M. Substrate-induced degradation of the $\alpha / \beta$-Fold hydrolase KARRIKIN INSENSITIVE2 requires a functional catalytic triad but is independent of MAX2. Mol Plant 8, 814-817 (2015).

68. Engler, C. et al. A golden gate modular cloning toolbox for plants. ACS Synth Biol 3, 839-843 (2014). alignment trimming in large-scale phylogenetic analyses. Bioinformatics 25, 1972-1973 (2009).

70. Letunic, I. \& Bork, P. Interactive Tree Of Life (iTOL): an online tool for phylogenetic tree 
display and annotation. Bioinformatics 23, 127-128 (2007).

600 71. Martinez-Trujillo, M., Limones-Briones, V., Cabrera-Ponce, J. L. \& Herrera-Estrella, L.

method. Plant Mol Biol Rep 22, 63-70 (2004).

72. Xu, Y. et al. Structural analysis of HTL and D14 proteins reveals the basis for ligand selectivity in Striga. Nat Commun 9, 3947 (2018).

\section{Acknowledgements}

We thank Prof. Yuichiro Tsuchiya (Institute of Transformative Bio-Molecules, Nagoya University),

609 Prof. Lam-Son Phan Tran (Institute of Genomics for Crop Abiotic Stress Tolerance, Texas Tech 610 University) and the late Prof. Kenji Mori for sharing L. philippensis seeds, A. thaliana d14 kai2 mutant 611 seeds and (+)-strigol, respectively. This work was supported by Ministry of Education, Culture, Sports, 612 Science and Technology KAKENHI grants (19K16169 to S.C., 20H05909 and 21H02506 to K.S. and 613 S.Y., 17H06172 to K.S); the Japan Society for the Promotion of Science (JSPS) KAKENHI Grants-in614 Aid for JSPS Fellows (JP21J00718 to S.O.); JST PRESTO (JPMJPR194D to S.Y.).

617 S.O., D.C.N., S.Y., and K.S. conceived and designed the study; K.S. supervised the experiments; S.O., 
618 S.C., and A.R.F.W. conducted the experiments; all authors analysed the data; S.O., and K.S. drafted

619 the original manuscript; all authors critically revised the manuscript and approved the final version.

621 Ethics declaration

622 The authors declare no competing interests.

623

624 Additional information

625 Supplementary information

626 Supplementary Figure 1: Chemical structure of SLs and synthetic analogues used in this study.

627 Supplementary Figure 2: Chemotropic response to rac-STR and YLG in non-parasitic plants.

628 Supplementary Figure 3: Chemotropic phenotype to $\mathrm{rac}$-STR on nutrient-containing media.

629 Supplementary Figure 4: Classification of KAI2 proteins in dicots.

630 Supplementary Figure 5: Alignment of KAI2d, KAI2i and KAI2c proteins in P. japonicum, KAI2d

631 in $S$. hermonthica, KAI2 and D14 in $A$. thaliana, and D14 and D14L in rice.

632 Supplementary Table 1: Chemicals used in this study.

633 Supplementary Table 2: Gene IDs and accession numbers of the

$634 \quad P$. japonicum genes investigated in this study.

635 Supplementary Table 3: Primers used in this study.

636 Supplementary Video 1 Time-lapse imaging of chemotropism to YLG in P. japonicum . 
637 Supplementary Video 2 Time-lapse imaging of tropism to rice in P. japonicum.

639 Supplementary Video 1 Time-lapse imaging of chemotropism to YLG in $P$. japonicum. Filter paper

640 disks were soaked in $1 \mu \mathrm{M}$ YLG (left) or 0.1\% (v/v) DMSO (right) and placed 5-mm from the root of

a 3-day old $P$. japonicum seedling growing on $0.7 \%$ INA agar medium $(\mathrm{w} / \mathrm{v})$. Images were taken for

18 hours at intervals of 648 seconds.

643

Supplementary Video 2 Time-lapse imaging of tropism to rice in $P$. japonicum. Root growth of $P$.

japonicum was captured for 48 hours at intervals of 20 minutes after being placed between the wild-

\section{Figure legends}

Fig.1: Chemotropic phenotype of Orobanchaceae parasitic plants to SLs and analogues. a-f,

652 Representative images of $P$. japonicum seedlings treated with $1 \mu \mathrm{M}$ chemical solutions diluted in $0.1 \%$

653 DMSO. Photos were taken 1 day after treatment. (a) 0.1\% (v/v) DMSO; (b) rac-STR; (c) rac-ORO;

654 (d) (+)-5DS; (e) rac-GR24; (f) YLG. g-h Percentage of P. japonicum plants that showed chemotropism

to each chemical. Three independent batches (3-12 and 4-8 seedlings in each batch for $\mathbf{g}$ and $\mathbf{h}$, 
656 respectively) for each compound. I, A representative image of $P$. japonicum exhibiting YLG-derived 657 fluorescence after treatment with a $100 \mu \mathrm{M}$ YLG solution. Filter paper disks were placed 5-mm to the 658 left of the roots. Confocal photos were taken 6 hours after treatment. j-k, Representative images of $S$. 659 hermonthica seedlings treated with $1 \mu \mathrm{M} r a c$-STR or $0.1 \%(\mathrm{v} / \mathrm{v})$ DMSO solutions. Photos were taken 6601 day after treatment. (j) $0.1 \%$ DMSO; (k) rac-STR. (I) Percentage of $S$. hermonthica plants that 661 showed chemotropism to $1 \mu \mathrm{M}$ rac-STR. Three or four independent batches (2-13 plants) were treated 662 with each compound. m, A representative image of S. hermonthica exhibiting YLG-derived 663 fluorescence when treated with a $100 \mu \mathrm{M}$ YLG solution. Filter paper disks were placed 3-mm to the 664 left of the roots. Confocal photos were taken 24 hours after treatment. n-p, Root chemotropism 665 root and a $d 10$ root that were aligned vertically at approximately 2-mm apart. Quantitative measurements (n,o) and images (p) were collected at 0,1 and 2 day(s) post co-incubation. (n) Time668 lapse growth of. P. japonicum roots. Plots indicate the positions of each root. (o) Horizontal distances 669 between $P$. japonicum root tips and rice roots. (p) Representative images of a $P$. japonicum root 670 growing towards WT and not towards $d 10$. Experiments were repeated three times with a total of 671 twenty-four P. japonicum seedlings. g,h,l Mean \pm standard error of the mean (SEM). Roots in which 672 growth had stopped were excluded from the calculation. i, m Arrowheads indicate asymmetrical 673 fluorescence. g,h Different letters indicate statistical significance at $P<0.05$ (two-way ANOVA, 674 Tukey's multiple comparison test). $\mathbf{l} * * P<0.01$ (Welch's $t$ test). Scale bars indicate $1 \mathrm{~mm}$ for $\mathbf{a}-\mathbf{f}, \mathbf{j}, \mathbf{k}$, 
$\mathbf{p}, 200 \mu \mathrm{m}$ for $\mathbf{i}$, and $100 \mu \mathrm{m}$ for $\mathbf{m}$, respectively.

Fig. 2: Chemotropic phenotype towards SLs on nutrient-containing media. a-d, Chemotropic

responses to $r a c$-STR in varying nutrient conditions in the presence of a $1 \mu \mathrm{M} r a c$-STR solution.

Photos were taken 1 day after treatment. (a) agar without any supplementary nutrients; (b) 1/2MS

(Nihon Pharmaceutical) with 1\%(w/v) sucrose (suc); (c) macronutrients of 1/2MS + suc; (d)

micronutrients of $1 / 2 \mathrm{MS}$ without suc. e, A representative image of $P$. japonicum plants showing YLG-

derived fluorescence when grown on $1 / 2 \mathrm{MS}+$ suc upon treatment with $100 \mu \mathrm{M}$ YLG solution. Filter

paper disks were placed 5-mm to the left of the roots. Confocal photos were taken 6 hours after treatment. The arrowhead indicates asymmetrical fluorescence. $\mathbf{f}-\mathbf{h}$, Percentage of $P$. japonicum

plants) were tested for each compound. Plants in which root growth had stopped were excluded from

and a reduced nitrogen source. $\mathrm{KCl}$ was added to adjust the potassium concentration. (h) Assays on

PjSMAX1.2 on agar without nutrients or 1/2MS + suc (4 technical replicates). (j) Expression level of 
694 of PjSMAX1.2 on 1/2MS + suc agar with a limited nitrogen source (3 technical replicates). f-k Mean

$695 \pm$ SEM. f, $\mathbf{h}, \mathbf{j}, \mathbf{k} * P<0.05,{ }^{* *} P<0.01, * * * P<0.001$ (Welch's $t$ test) in comparison with the no

696 nutrient treatment. g Different letters indicate a statistical significance at $P<0.05$ (two-way ANOVA,

697 Tukey's multiple comparison test). $\mathbf{i} * * P<0.01, * * * P<0.001$ (Welch's $t$ test). Scale bars indicate 1

$698 \mathrm{~mm}$ for a-d and $200 \mu \mathrm{m}$ for e, respectively.

Fig. 3: Expression dynamics of auxin-related genes upon SL treatment. Expression patterns of the

DR5 (a-h) and PjPIN2 (i-p) promoters driving expression of a fluorescent marker gene upon treatment

with $1 \mu \mathrm{M} \mathrm{rac}$-STR or $0.1 \%$ (v/v) DMSO solutions. Filter paper disks were placed 5-mm to the left of

the roots. Confocal photos were taken at the indicated time points after treatment on media with

$(1 / 2 \mathrm{MS}+$ suc) or without (agar) nutrient. Bright-field and fluorescent images were merged in a-h. Venus

fluorescence intensity was represented in a Rainbow RGB spectrum in i-p using Image ${ }^{66}$. White ovals

indicate asymmetrical expression. The scale bars in $\mathbf{a}$ and $\mathbf{i}$ also refer to $\mathbf{b}-\mathbf{h}$ and $\mathbf{j}-\mathbf{p}$, respectively. Bars

$=100 \mu \mathrm{m}$.

Fig. 4: Analyses of KAI2 homologs in P. japonicum. a, KAI2d phylogeny. The KAI2i clade was

710 included as an outgroup. Clades are coloured blue, green, and red for facultative hemiparasites,

711 obligate hemiparasite, and obligate holoparasites, respectively. Purple and yellow words indicate

712 KAI2d forms in S. hermonthica that recognise SLs ${ }^{30,72}$ and KAI2d forms in P. japonicum (PjKAI2d), 
713 respectively. Coloured boxes indicate gene expression levels of PjKAI2d in roots or S. hermonthica

$714 K A I 2 d$ in seeds, seedlings, or rice plants at 1-, 3-, 7-day post infection (dpi $)^{18,29}$. The bar indicates

715 substitutions per site. Bootstrap values are indicated at the nodes. b, Relative expression levels of

716 PjKAI2 genes. Representative data are shown (4 technical replicates) using PjUBC2 as the reference

717 gene. Experiments were performed three times with similar results. c, Complementation assays for

718 PjKAI2d. Arabidopsis kai2 d14 (Col-0 ecotype) was used for the null mutant to express PjKAI2d

719 under control of the AtKAI2 promoter. Germination rates were calculated 5 days after incubation at

$72022^{\circ} \mathrm{C}$ in the dark. Representative data are shown (4 independent batches, $23-87$ seeds per batch). d-e,

721 Chemotropic responses to rac-STR in PjKAI2d2 dominant negative plants. (d) Percentage of $P$.

722 japonicum transgenic hairy roots that were chemotropic to $1 \mu \mathrm{M} \mathrm{rac}$-STR or $0.1 \%(\mathrm{v} / \mathrm{v}) \mathrm{DMSO}$. Three

723 independent batches ( 2 to 6 plants) for each compound. Plants that stopped root growth were excluded

724 from the calculations. (e) Relative expression level of PjSMAX1.2. Representative data are shown (4

725 technical replicates) using $P j U B C 2$ as the reference gene. Experiments were performed three times

726 with similar results. b-e Mean \pm SEM. b,d,e $* \mathrm{P}<0.05, * * P<0.01, * * * P<0.001$ (Welch's $t$ test). c

$727 * P<0.05, * * P<0.01,{ }^{* * *} P<0.001$ (Welch's $t$ test), in comparison to DMSO treatment for each

728 genotype. 

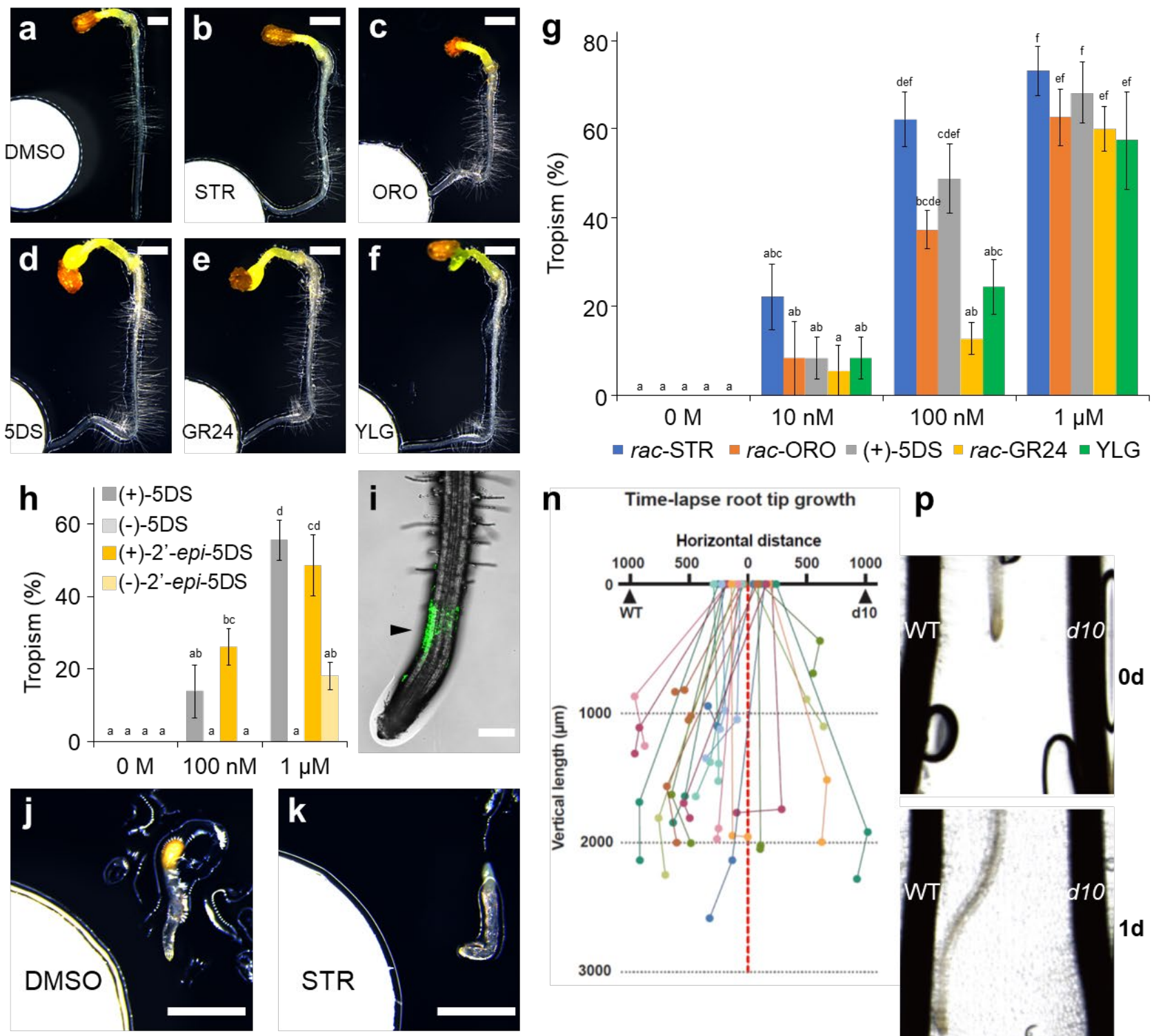

$\mathbf{n}$

Time-lapse root tip growth $\mathbf{p}$
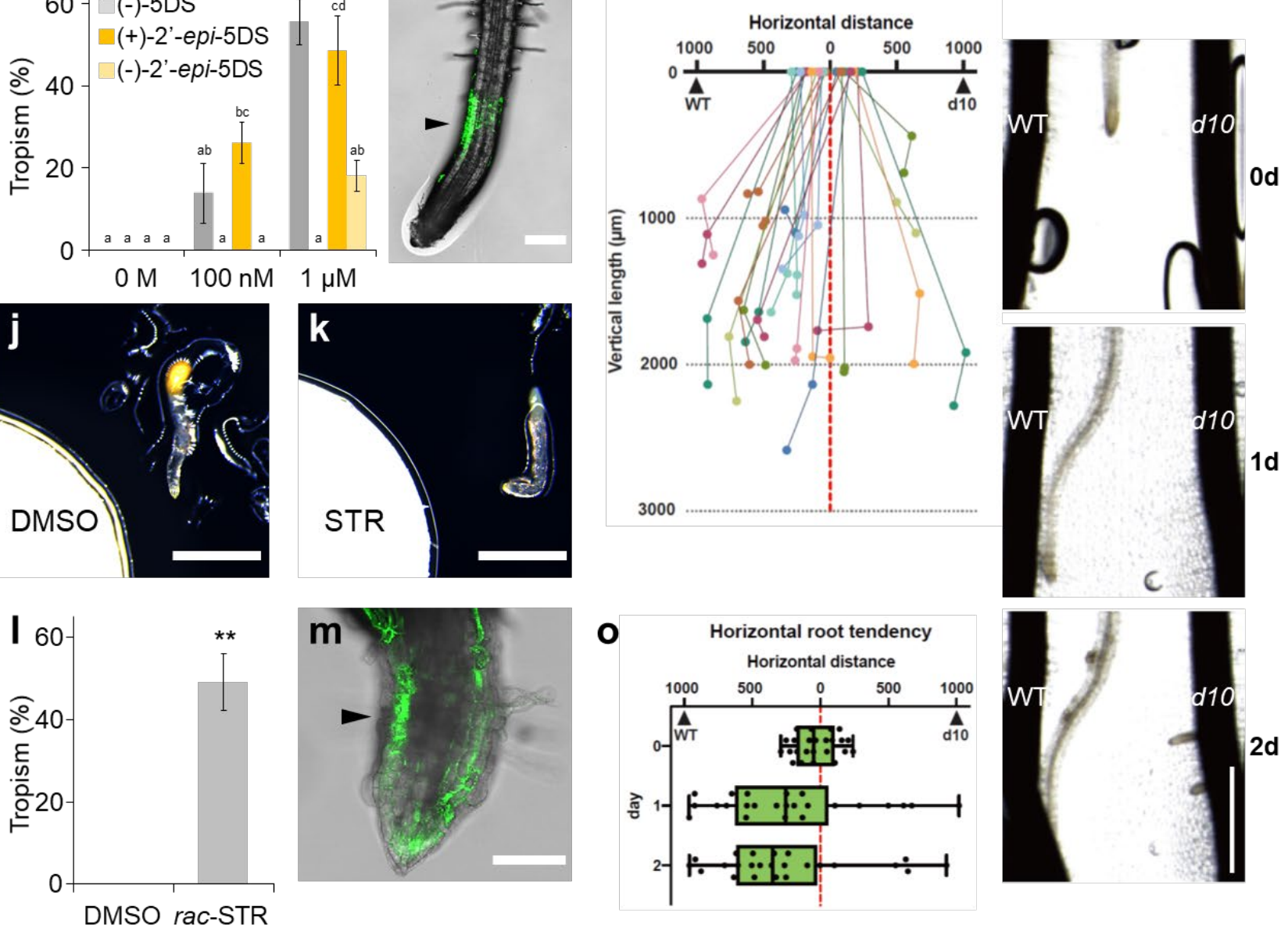

$\mathbf{0}$
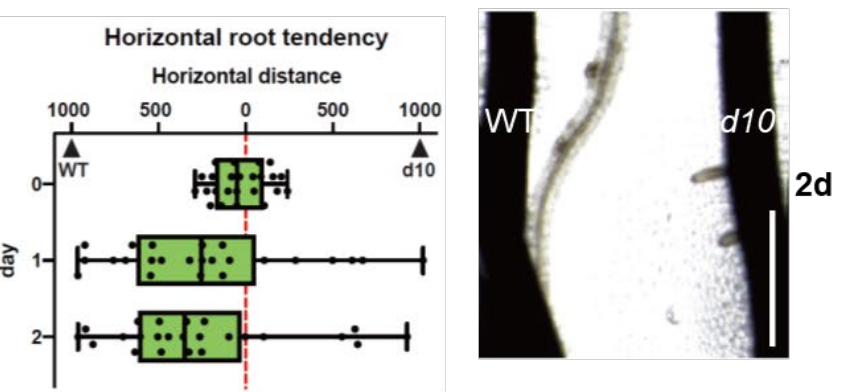


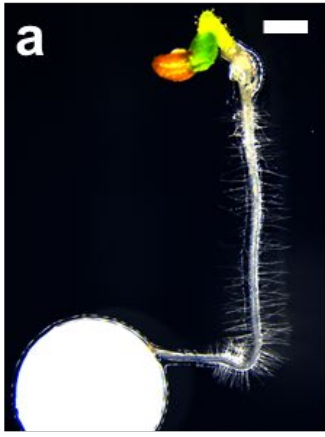

Agar

f

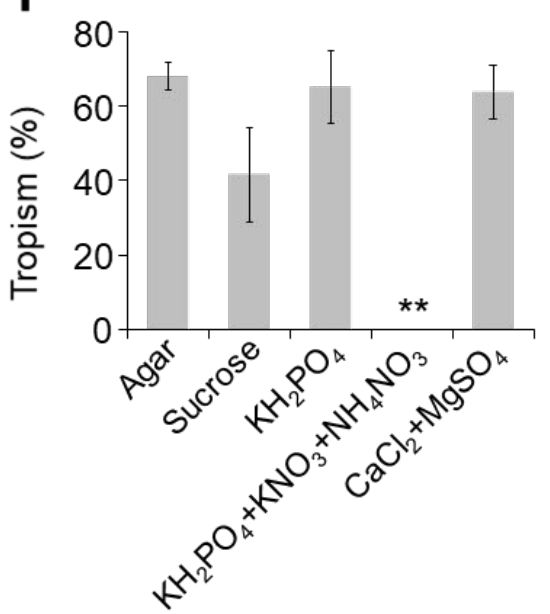

i

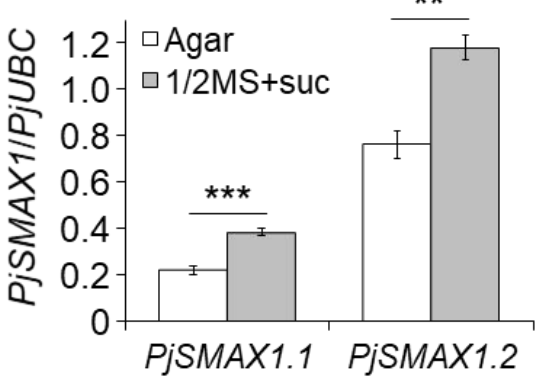

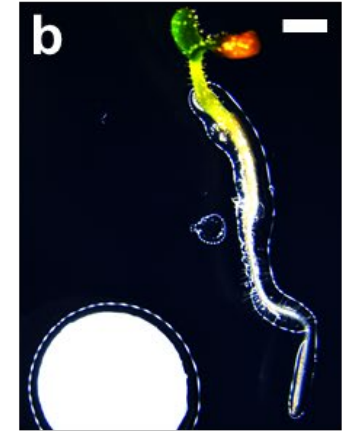

$1 / 2 \mathrm{MS}+$ suc

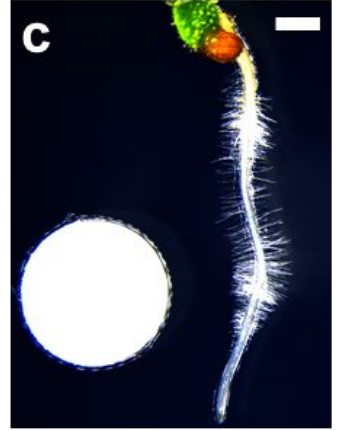

Macronutrients+suc

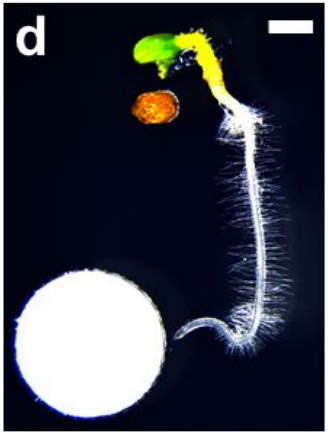

Micronutrients

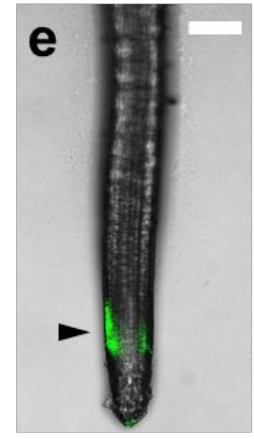

$1 / 2 \mathrm{MS}+$ suc g
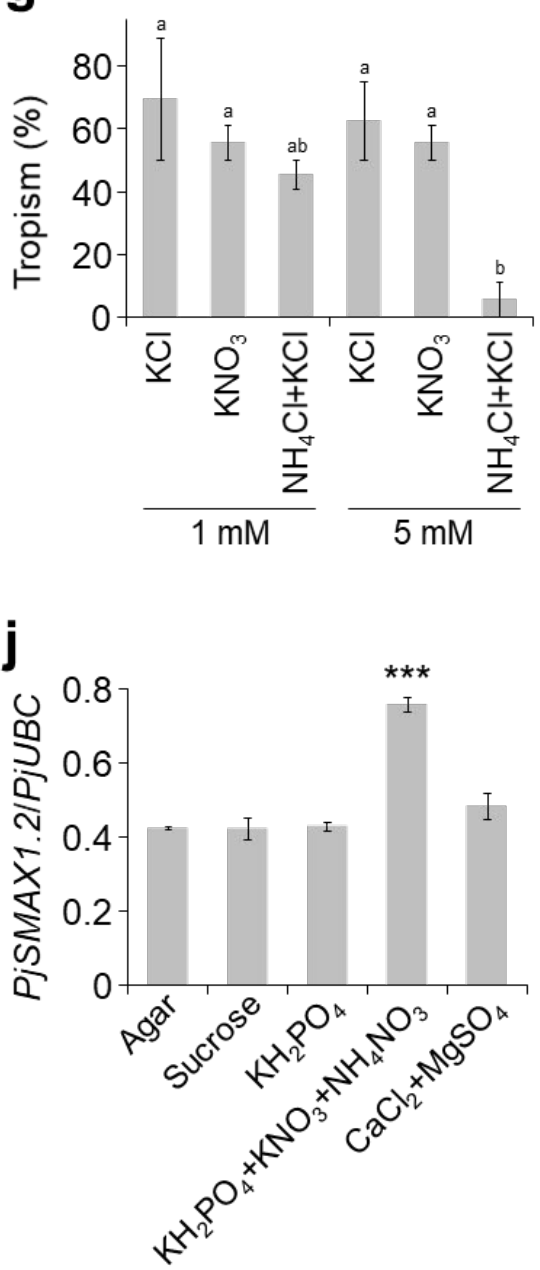

h

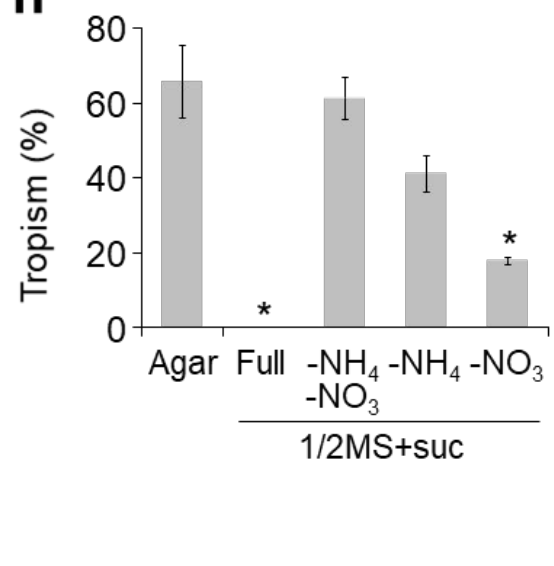

k

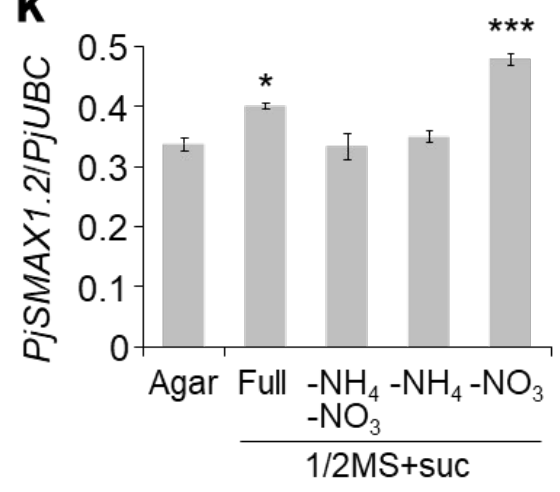


Figure 3

737

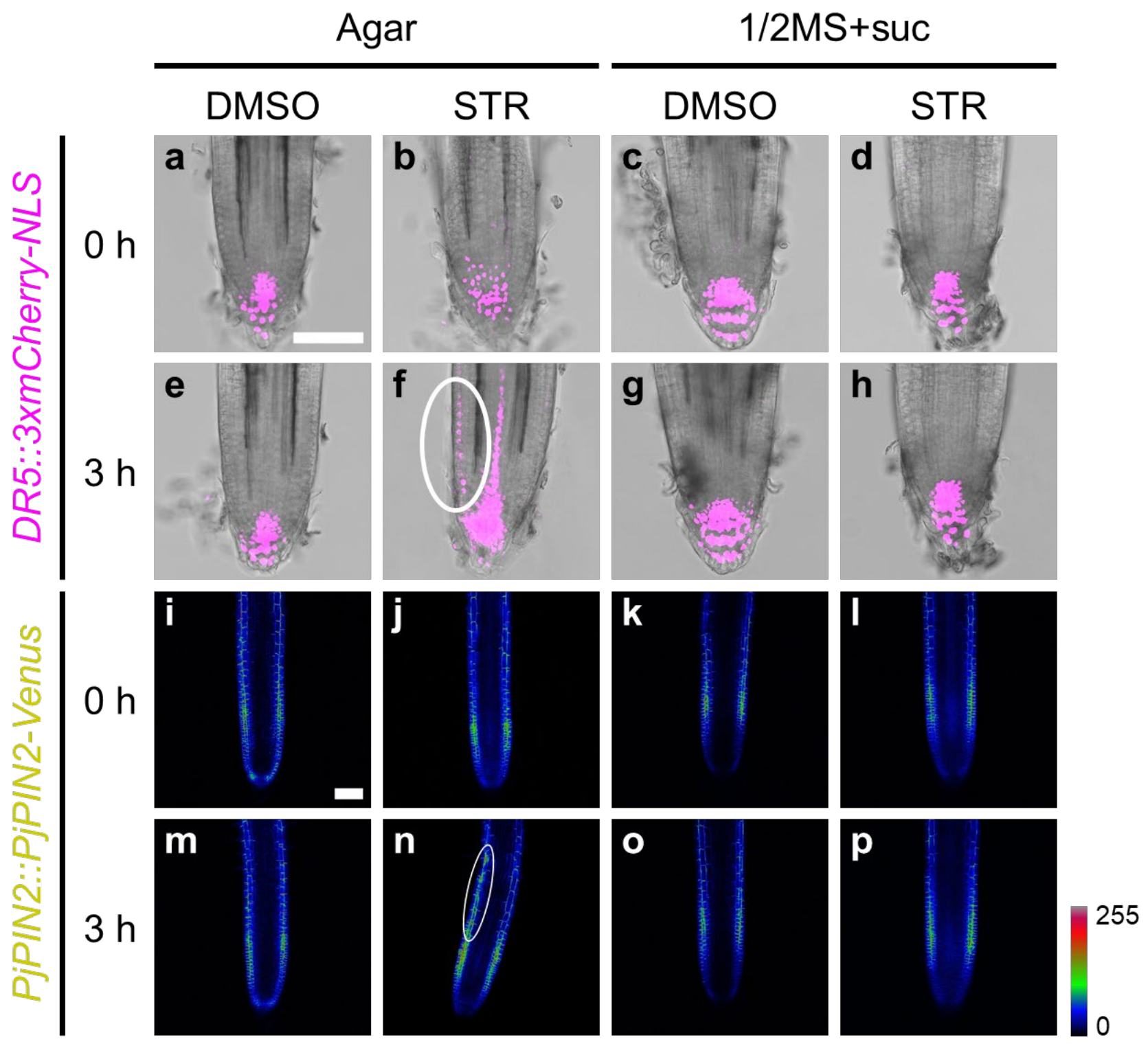


bioRxiv preprint doi: https://doi.org/10.1101/2022.02.17.480806; this version posted February 19, 2022. The copyright holder for this

a
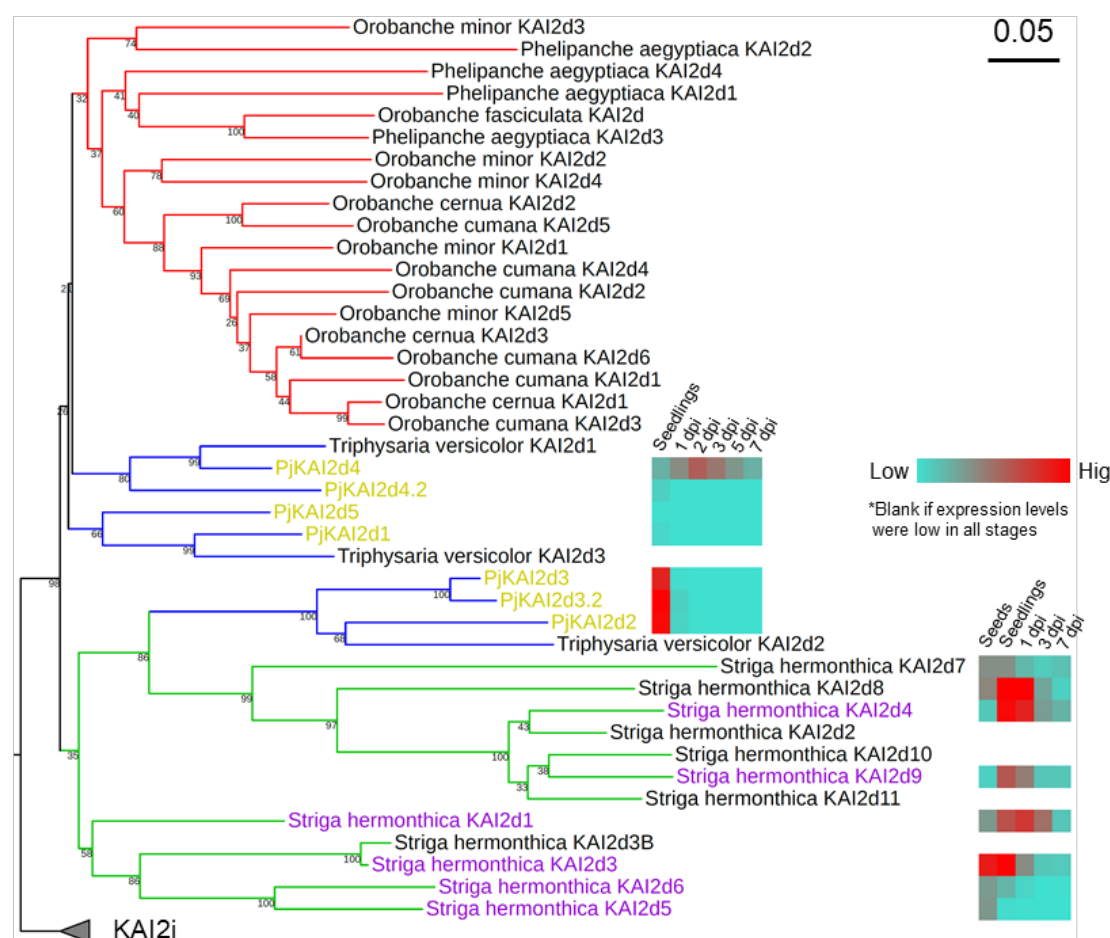

b

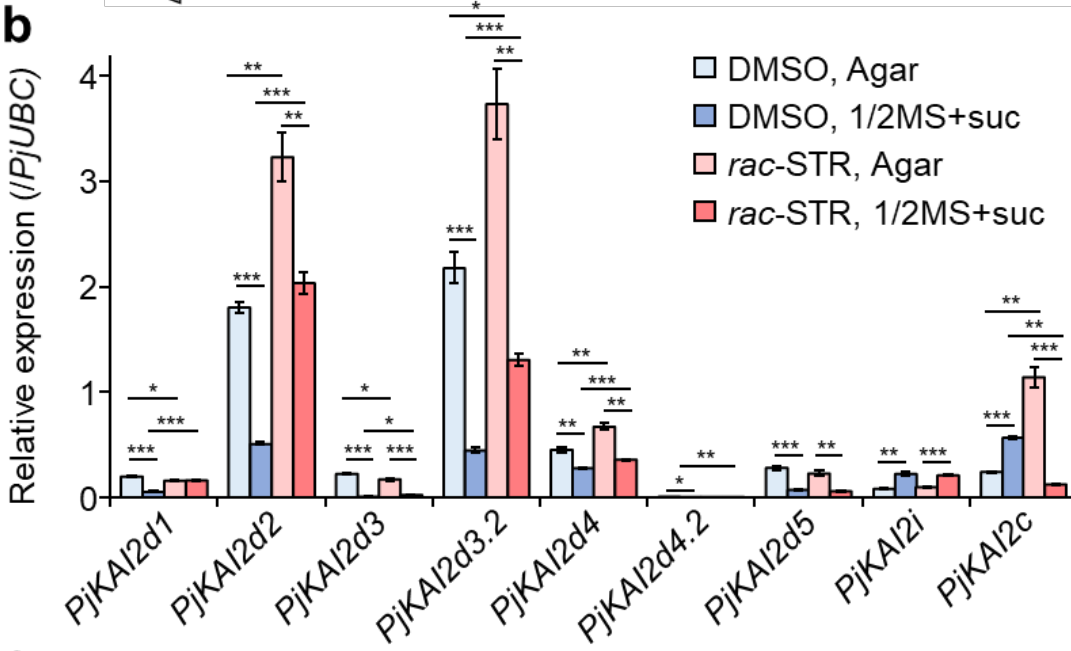

C

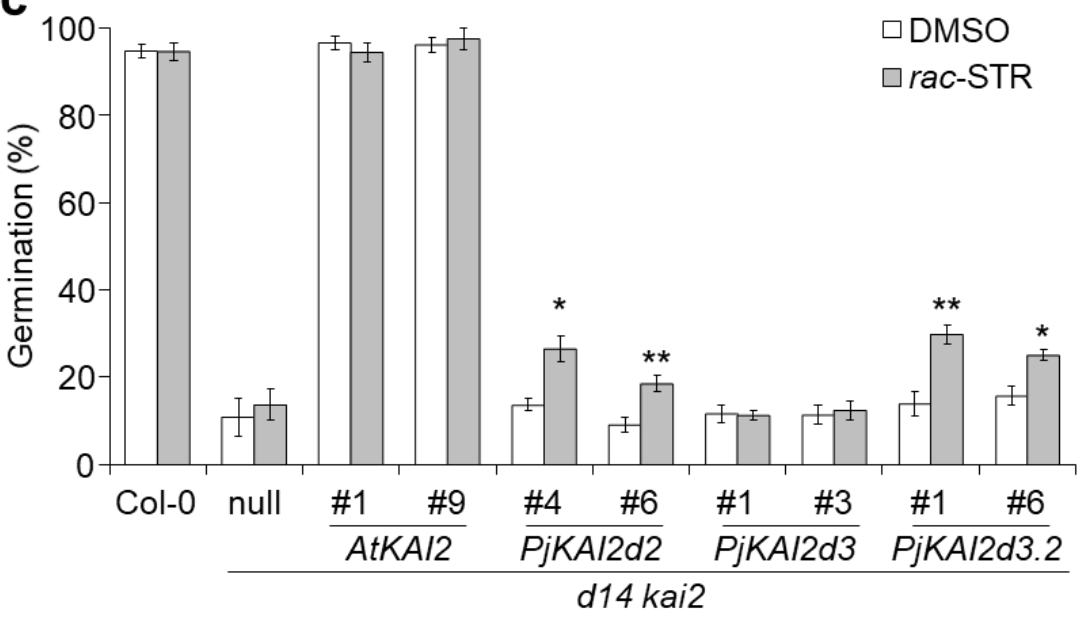

e
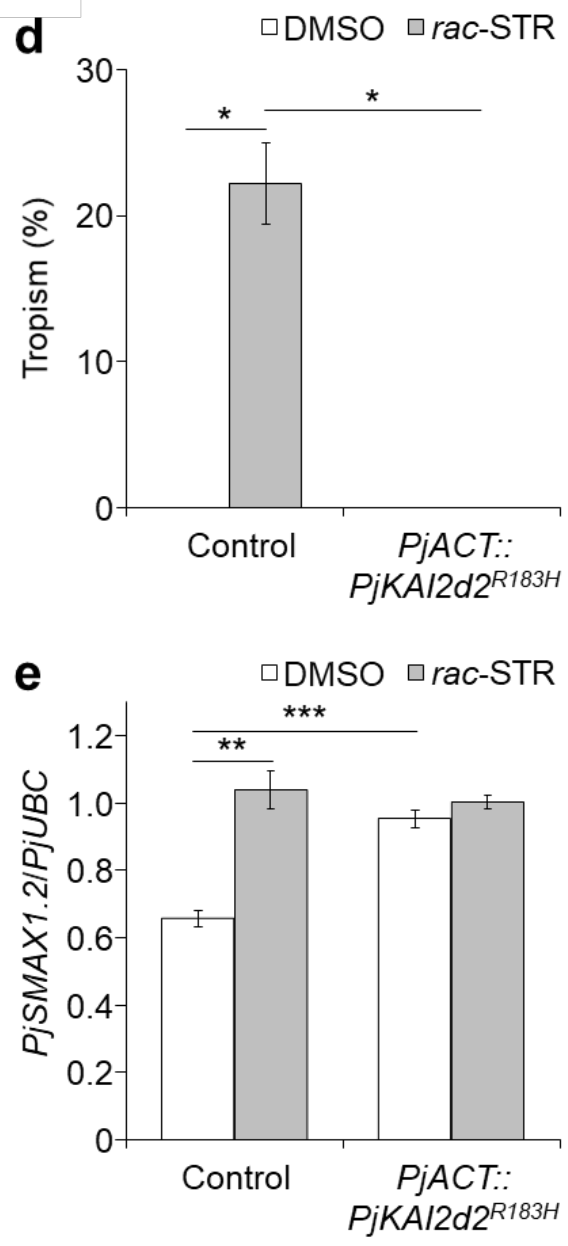